\title{
Covariant Hamiltonian Field Theory. Path Integral Quantization
}

\author{
D. Bashkirov ${ }^{1}$ and G. Sardanashvily ${ }^{2}$ \\ Department of Theoretical Physics, Physics Faculty, Moscow State University, 117234 Moscow, \\ Russia
}

The Hamiltonian counterpart of classical Lagrangian field theory is covariant Hamiltonian field theory where momenta correspond to derivatives of fields with respect to all world coordinates. In particular, classical Lagrangian and covariant Hamiltonian field theories are equivalent in the case of a hyperregular Lagrangian, and they are quasi-equivalent if a Lagrangian is almost-regular. In order to quantize covariant Hamiltonian field theory, one usually attempts to construct and quantize a multisymplectic generalization of the Poisson barcket. In the present work, the path integral quantization of covariant Hamiltonian field theory is suggested. We use the fact that a covariant Hamiltonian field system is equivalent to a certain Lagrangian system on a phase space which is quantized in the framework of perturbative quantum field theory. We show that, in the case of almost-regular quadratic Lagrangians, path integral quantizations of associated Lagrangian and Hamiltonian field systems are equivalent.

\section{INTRODUCTION}

As is well-known, the familiar symplectic Hamiltonian technique applied to field theory leads to instantaneous Hamiltonian formalism on an infinite-dimensional phase space coordinated by field functions at some instant of time (see (Gotay, 1991) for the strict mathematical exposition of this formalism). The true Hamiltonian counterpart of classical first-order Lagrangian field theory is covariant Hamiltonian formalism, where canonical momenta $p_{i}^{\mu}$ correspond to derivatives $y_{\mu}^{i}$ of fields $y^{i}$ with respect to all world coordinates $x^{\mu}$. This formalism has been vigorously developed since the 1970s in its polysymplectic, multisymplectic and Hamilton - De Donder variants (see (Giachetta et al., 1997, 1999; Echeverría-Enríquez et al., 2000; Hélein and Kouneiher, 2002; Echeverría-Enríquez et al., 2004) and references therein). In order to quantize covariant Hamiltonian field theory, one usually attempts to

\footnotetext{
${ }^{1}$ E-mail: bashkird@rol.ru

${ }^{2}$ E-mail: sard@grav.phys.msu.su; Web: http://webcenter.ru/ sardan/
} 
construct multisymplectic generalization of the Poisson barcket with respect to the derivatives $\partial / \partial y^{i}$ and $\partial / \partial p_{i}^{\mu}$ (Kanatchikov, 1999; Castrillón Lòpez and Marsden, 2003; Forger et al., 2003).

We have suggested to quantize covariant (polysymplectic) Hamiltonian field theory in path integral terms (Sardanashvily, 1994). In the present work, this quantization scheme is modified owing to the fact that a polysymplectic Hamiltonian system with a Hamiltonian $\mathcal{H}\left(x^{\mu}, y^{i}, p_{i}^{\mu}\right)$ is equivalent to a Lagrangian system with the Lagrangian

$$
\mathcal{L}_{\mathcal{H}}\left(x^{\mu}, y^{i}, p_{i}^{\mu}, y_{\lambda}^{i}\right)=p_{i}^{\lambda} y_{\lambda}^{i}-\mathcal{H}\left(x^{\mu}, y^{i}, p_{i}^{\mu}, y_{\lambda}^{i}\right)
$$

of the variables $y^{i}$ and $p_{i}^{\mu}$. This Lagrangian system can be quantized in the framework of familiar perturbative quantum field theory. If there is no constraint and the matrix $\partial^{2} \mathcal{H} / \partial p_{i}^{\mu} \partial p_{j}^{\nu}$ is nondegenerate and positive-definite, this quantization is given by the generating functional

$$
Z=\mathcal{N}^{-1} \int \exp \left\{\int\left(\mathcal{L}_{\mathcal{H}}+\Lambda+i J_{i} y^{i}+i J_{\mu}^{i} p_{i}^{\mu}\right) d x\right\} \prod_{x}[d p(x)][d y(x)]
$$

of Euclidean Green functions, where $\Lambda$ comes from the normalization condition

$$
\int \exp \left\{\int\left(-\frac{1}{2} \partial_{\mu}^{i} \partial_{\nu}^{j} \mathcal{H} p_{i}^{\mu} p_{j}^{\nu}+\Lambda\right) d x\right\} \prod_{x}[d p(x)]=1 .
$$

If a Hamiltonian $\mathcal{H}$ is degenerate, the Lagrangian $\mathcal{L}_{\mathcal{H}}(1)$ may admit gauge symmetries. In this case, integration of a generating functional along gauge group orbits must be finite. If there are constraints, the Lagrangian system with a Lagrangian $\mathcal{L}_{\mathcal{H}}(1)$ restricted to the constraint manifold is quantized.

In order to verify this path quantization scheme, we apply it to Hamiltonian field systems associated to Lagrangian field systems with quadratic Lagrangians

$$
\mathcal{L}=\frac{1}{2} a_{i j}^{\lambda \mu} y_{\lambda}^{i} y_{\mu}^{j}+b_{i}^{\lambda} y_{\lambda}^{i}+c
$$

where $a, b$ and $c$ are functions of world coordinates $x^{\mu}$ and field variables $y^{i}$. Note that, in the framework of perturbative quantum field theory, any Lagrangian is split into the sum of a quadratic Lagrangian (3) and an interaction term quantized as a perturbation.

For instance, let the Lagrangian (3) be hyperregular, i.e., the matrix function $a$ is nondegenerate. Then there exists a unique associated Hamiltonian system whose Hamiltonian $\mathcal{H}$ is quadratic in momenta $p_{i}^{\mu}$, and so is the Lagrangian $\mathcal{L}_{\mathcal{H}}(1)$. If the matrix function $a$ is positive-definite on an Euclidean space-time, the generating functional (2) is a Gaussian 
integral of momenta $p_{i}^{\mu}(x)$. Integrating $Z$ with respect to $p_{i}^{\mu}(x)$, one restarts the generating functional of quantum field theory with the original Lagrangian $\mathcal{L}(3)$. We extend this result to field theories with almost-regular Lagrangians $\mathcal{L}(3)$, e.g., Yang-Mills gauge theory. The key point is that, though such a Lagrangian $\mathcal{L}$ yields constraints and admits different associated Hamiltonians $\mathcal{H}$, all the Lagrangians $\mathcal{L}_{\mathcal{H}}$ coincide on the constraint manifold, and we have a unique constrained Hamiltonian system which is quasi-equivalent to the original Lagrangian one (Giachetta at al., 1997, 1999).

\section{COVARIANT HAMILTONIAN FIELD THEORY}

We follow the geometric formulation of classical field theory where classical fields are represented by sections of fiber bundles. Let $Y \rightarrow X$ be a smooth fiber bundle provided with bundle coordinates $\left(x^{\mu}, y^{i}\right)$. The configuration space of Lagrangian field theory on $Y$ is the first-order jet manifold $J^{1} Y$ of $Y$. It is equipped with the bundle coordinates $\left(x^{\mu}, y^{i}, y_{\mu}^{i}\right)$ compatible with the composite fibration

$$
J^{1} Y \stackrel{\pi_{0}^{1}}{\longrightarrow} Y \stackrel{\pi}{\longrightarrow} X
$$

Any section $s$ of $Y \rightarrow X$ is prolonged to the section $J^{1} s$ of $J^{1} Y \rightarrow X$ such that $y_{\mu}^{i} \circ J^{1} s=\partial_{\mu} s^{i}$. A first-order Lagrangian is defined as a horizontal density

$$
L=\mathcal{L} \omega: J^{1} Y \rightarrow \wedge^{n} T^{*} X, \quad \omega=d x^{1} \wedge \cdots d x^{n}, \quad n=\operatorname{dim} X
$$

on the jet manifold $J^{1} Y$. The corresponding Euler-Lagrange equations are given by the subset

$$
\left(\partial_{i}-d_{\lambda} \partial_{i}^{\lambda}\right) \mathcal{L}=0, \quad d_{\lambda}=\partial_{\lambda}+y_{\lambda}^{i} \partial_{i}+y_{\lambda \mu}^{i} \partial_{i}^{\mu},
$$

of the second-order jet manifold $J^{2} Y$ of $Y$ coordinated by $\left(x^{\mu}, y^{i}, y_{\lambda}^{i}, y_{\lambda \mu}^{i}\right)$. A section $s$ of $Y \rightarrow X$ is a solution of these equations if its second jet prolongation $J^{2} s$ lives in the subset (5).

The phase space of covariant (polysymplectic) Hamiltonian field theory on $Y$ is the Legendre bundle

$$
\Pi=\wedge_{\wedge}^{n} T^{*} X \underset{Y}{\otimes} V^{*} Y \underset{Y}{\otimes} T X=V^{*} Y \wedge\left(\wedge^{n-1} T^{*} X\right),
$$

where $V^{*} Y$ is the vertical cotangent bundle of $Y \rightarrow X$. The Legendre bundle $\Pi$ is equipped with the holonomic bundle coordinates $\left(x^{\lambda}, y^{i}, p_{i}^{\mu}\right)$ compatible with the composite fibration

$$
\Pi \stackrel{\pi_{Y}}{\longrightarrow} Y \stackrel{\pi}{\longrightarrow} X
$$


It is endowed with the canonical polysymplectic form

$$
\Omega=d p_{i}^{\lambda} \wedge d y^{i} \wedge \omega \otimes \partial_{\lambda}
$$

A covariant Hamiltonian $\mathcal{H}$ on $\Pi(6)$ is defined as a section $p=-\mathcal{H}$ of the trivial onedimensional fiber bundle

$$
Z_{Y}=T^{*} Y \wedge\left(\stackrel{n-1}{\wedge} T^{*} X\right) \rightarrow \Pi
$$

equipped with holonomic bundle coordinates $\left(x^{\lambda}, y^{i}, p_{i}^{\mu}, p\right)$. This fiber bundle is provided with the canonical multisymplectic Liouville form

$$
\left.\Xi=p \omega+p_{i}^{\lambda} d y^{i} \wedge \omega_{\lambda}, \quad \omega_{\lambda}=\partial_{\lambda}\right\rfloor \omega
$$

The pull-back of $\Xi$ onto $\Pi$ by a Hamiltonian $\mathcal{H}$ is a Hamiltonian form

$$
H=\mathcal{H}^{*} \Xi_{Y}=p_{i}^{\lambda} d y^{i} \wedge \omega_{\lambda}-\mathcal{H} \omega
$$

on $\Pi$. The corresponding covariant Hamilton equations on $\Pi$ are given by the closed submanifold

$$
y_{\lambda}^{i}=\partial_{\lambda}^{i} \mathcal{H}, \quad p_{\lambda i}^{\lambda}=-\partial_{i} \mathcal{H}
$$

of the jet manifold $J^{1} \Pi$ of $\Pi$. A section $r$ of $\Pi \rightarrow X$ is a solution of these equations if its jet prolongation $J^{1} r$ lives in the submanifold (9).

Proposition 1. A section $r$ of $\Pi \rightarrow X$ is a solution of the covariant Hamilton equations (9) iff it satisfies the condition $\left.r^{*}(u\rfloor d H\right)=0$ for any vertical vector field $u$ on $\Pi \rightarrow X$.

Proposition 2. A section $r$ of $\Pi \rightarrow X$ is a solution of the covariant Hamilton equations (9) iff it is a solution of the Euler-Lagrange equations for the first-order Lagrangian

$$
L_{\mathcal{H}}=h_{0}(H)=\mathcal{L}_{\mathcal{H}} \omega=\left(p_{i}^{\lambda} y_{\lambda}^{i}-\mathcal{H}\right) \omega
$$

on $J^{1} \Pi$, where $h_{0}$ sends exterior forms on $\Pi$ onto horizontal exterior forms on $J^{1} \Pi \rightarrow X$ by the rule $h_{0}\left(d y^{i}\right)=y_{\lambda}^{i} d x^{\lambda}$.

Note that, for any section $r$ of $\Pi \rightarrow X$, the pull-backs $r^{*} H$ and $J^{1} r^{*} L_{\mathcal{H}}$ coincide. This fact and Proposition 2 motivate us to quantize covariant Hamiltonian field theory with a Hamiltonian $\mathcal{H}$ on $\Pi$ as a Lagrangian system with the Lagrangian $L_{\mathcal{H}}(10)$.

Furthermore, let $i_{N}: N \rightarrow \Pi$ be a closed imbedded subbundle of the Legendre bundle $\Pi \rightarrow Y$ which is regarded as a constraint space of a covariant Hamiltonian field system with a Hamiltonian $\mathcal{H}$. This Hamiltonian system is restricted to $N$ as follows. Let $H_{N}=i_{N}^{*} H$ 
be the pull-back of the Hamiltonian form $H$ (8) onto $N$. The constrained Hamiltonian form $H_{N}$ defines the constrained Lagrangian

$$
L_{N}=h_{0}\left(H_{N}\right)=\left(J^{1} i_{N}\right)^{*} L_{\mathcal{H}}
$$

on the jet manifold $J^{1} N_{L}$ of the fiber bundle $N_{L} \rightarrow X$. The Euler-Lagrange equations for this Lagrangian are called the constrained Hamilton equations.

Note that, in fact, the Lagrangian $L_{\mathcal{H}}(10)$ is the pull-back onto $J^{1} \Pi$ of the horizontal form $L_{\mathcal{H}}$ on the bundle product $\Pi \underset{Y}{\times} J^{1} Y$ over $Y$ by the canonical map $J^{1} \Pi \rightarrow \Pi \underset{Y}{\times} J^{1} Y$. Therefore, the constrained Lagrangian $L_{N}$ (11) is simply the restriction of $L_{\mathcal{H}}$ to $N \underset{Y}{\times} J^{1} Y$. Proposition 3. A section $r$ of the fiber bundle $N \rightarrow X$ is a solution of constrained Hamilton equations iff it satisfies the condition $\left.r^{*}\left(u_{N}\right\rfloor d H\right)=0$ for any vertical vector field $u_{N}$ on $N \rightarrow X$.

It follows from Proposition 1 and Proposition 3 that any solution of the covariant Hamilton equations (9) which lives in the constraint manifold $N$ is also a solution of the constrained Hamilton equations on $N$. This fact motivates us to quantize covariant Hamiltonian field theory on a constraint manifold $N$ as a Lagrangian system with the pull-back Lagrangian $L_{N}(11)$.

Since a constraint manifold is assumed to be a closed imbedded submanifold of $\Pi$, there exists its open neighbourhood $U$ which is a fibered manifold $U \rightarrow N$. If $\Pi$ is a fibered manifold $\pi_{N}: \Pi \rightarrow N$ over $N$, it is often convenient to quantize a Lagrangian system on $\Pi$ with the pull-back Lagrangian $\pi_{N}^{*} L_{N}$, but integration of the corresponding generating functional along the fibers of $\Pi \rightarrow N$ must be finite.

In order to verify this quantization scheme, let us associate to a Lagrangian field system on $Y$ a covarinat Hamiltonian system on $\Pi$, then let us quantize this Hamiltonian system and compare this quantization with that of an original Lagrangian system.

\section{ASSOCIATED LAGRANGIAN AND HAMILTONIAN SYS- TEMS}

In order to relate classical Lagrangian and covariant Hamiltonian field theories, let us recall that, besides the Euler-Lagrange equations, a Lagrangian $L$ (4) also yields the Cartan equations which are given by the subset

$$
\begin{aligned}
& \left(\bar{y}_{\mu}^{j}-y_{\mu}^{j}\right) \partial_{i}^{\lambda} \partial_{j}^{\mu} \mathcal{L}=0, \quad \partial_{i} \mathcal{L}-\bar{d}_{\lambda} \partial_{i}^{\lambda} \mathcal{L}+\left(\bar{y}_{\mu}^{j}-y_{\mu}^{j}\right) \partial_{i} \partial_{j}^{\mu} \mathcal{L}=0, \\
& \bar{d}_{\lambda}=\partial_{\lambda}+\bar{y}_{\lambda}^{i} \partial_{i}+\bar{y}_{\lambda \mu}^{i} \partial_{i}^{\mu}
\end{aligned}
$$


of the repeated jet manifold $J^{1} J^{1} Y$ coordinated by $\left(x^{\mu}, y^{i}, y_{\lambda}^{i}, \bar{y}_{\lambda}^{i}, \bar{y}_{\lambda \mu}^{i}\right)$. A solution of the Cartan equations is a section $\bar{s}$ of the jet bundle $J^{1} Y \rightarrow X$ whose jet prolongation $J^{1} \bar{s}$ lives in the subset (12). Every solution $s$ of the Euler-Lagrange equations (5) defines the solution $J^{1} s$ of the Cartan equations (12). If $\bar{s}$ is a solution of the Cartan equations and $\bar{s}=J^{1} s$, then $s$ is a solution of the Euler-Lagrange equations. If a Lagrangian $L$ is regular, the equations (5) and (12) are equivalent.

Any Lagrangian $L(4)$ yields the Legendre map

$$
\widehat{L}: J^{1} Y \underset{Y}{\longrightarrow} \Pi, \quad p_{i}^{\lambda} \circ \widehat{L}=\partial_{i}^{\lambda} \mathcal{L}
$$

over Id $Y$ whose image $N_{L}=\widehat{L}\left(J^{1} Y\right)$ is called the Lagrangian constraint space. A Lagrangian $L$ is said to be hyperregular if the Legendre map (13) is a diffeomorphism. A Lagrangian $L$ is called almost-regular if the Lagrangian constraint space is a closed imbedded subbundle $i_{N}$ : $N_{L} \rightarrow \Pi$ of the Legendre bundle $\Pi \rightarrow Y$ and the surjection $\widehat{L}: J^{1} Y \rightarrow N_{L}$ is a submersion (i.e., a fibered manifold) whose fibers are connected. Conversely, any Hamiltonian $\mathcal{H}$ yields the Hamiltonian map

$$
\widehat{H}: \Pi \underset{Y}{\longrightarrow} J^{1} Y, \quad y_{\lambda}^{i} \circ \widehat{H}=\partial_{\lambda}^{i} \mathcal{H} .
$$

A Hamiltonian $\mathcal{H}$ on $\Pi$ is said to be associated to a Lagrangian $L$ on $J^{1} Y$ if $\mathcal{H}$ satisfies the relations

$$
\begin{array}{ll}
\widehat{L} \circ \widehat{H} \circ \widehat{L}=\widehat{L}, & p_{i}^{\mu}=\partial_{i}^{\mu} \mathcal{L}\left(x^{\mu}, y^{i}, \partial_{\lambda}^{j} \mathcal{H}\right), \quad\left(x^{\mu}, y^{i}, p_{i}^{\mu}\right) \in N_{L}, \\
\widehat{H}^{*} L_{\mathcal{H}}=\widehat{H}^{*} L, & p_{i}^{\mu} \partial_{\mu}^{i} \mathcal{H}-\mathcal{H}=\mathcal{L}\left(x^{\mu}, y^{j}, \partial_{\lambda}^{j} \mathcal{H}\right) .
\end{array}
$$

If an associated Hamiltonian $\mathcal{H}$ exists, the Lagrangian constraint space $N_{L}$ is given by the coordinate relations (15) and $\widehat{H} \circ \widehat{L}$ is a projector from $\Pi$ onto $N_{L}$.

For instance, any hyperregular Lagrangian $L$ admits a unique associated Hamiltonian $\mathcal{H}$ such that

$$
\widehat{H}=\widehat{L}^{-1}, \quad \mathcal{H}=p_{i}^{\mu} \widehat{L}_{\mu}^{-1 i}-\mathcal{L}\left(x^{\lambda}, y_{\lambda}^{i}, \widehat{L}_{\lambda}^{-1 i}\right) .
$$

In this case, any solution $s$ of the Euler-Lagrange equations (5) defines the solution $r=$ $\widehat{L} \circ J^{1} s$, of the covariant Hamilton equations (9). Conversely, any solution $r$ of these Hamilton equations yields the solution $s=\pi_{Y} \circ r$ of the Euler-Lagrange equations (5).

A degenerate Lagrangian need not admit an associated Hamiltonian. If such a Hamiltonian exists, it is not necessarily unique. Let us restrict our consideration to almost-regular Lagrangians. From the physical viewpoint, the most of Lagrangian field theories is of this 
type. From the mathematical one, this notion of degeneracy is particularly appropriate for the study of relations between Lagrangian and covariant Hamiltonian formalisms as follows.

Theorem 4. Let $L$ be an almost-regular Lagrangian and $\mathcal{H}$ an associated Hamiltonian. Let a section $r$ of $\Pi \rightarrow X$ be a solution of the covariant Hamilton equations (9) for $\mathcal{H}$. If $r$ lives in the constraint manifold $N_{L}$, then $s=\pi_{Y} \circ r$ satisfies the Euler-Lagrange equations (5) for $L$, while $\bar{s}=\widehat{H} \circ r$ obeys the Cartan equations (12). Conversely, let $\bar{s}$ be a solution of the Cartan equations (12) for $L$. If $\mathcal{H}$ satisfies the relation

$$
\widehat{H} \circ \widehat{L} \circ \bar{s}=J^{1}\left(\pi_{0}^{1} \circ \bar{s}\right)
$$

the section $r=\widehat{L} \circ \bar{s}$ of the Legendre bundle $\Pi \rightarrow X$ is a solution of the Hamilton equations (9) for $\mathcal{H}$. If $\bar{s}=J^{1} s$, we obtain the relation between solutions the Euler-Lagrange equations and the covariant Hamilton ones.

By virtue of Theorem 4, one need a set of different associated Hamiltonians in order to recover all solutions of the Euler-Lagrange and Cartan equations for an almost-regular Lagrangian $L$. We can overcome this ambiguity as follows.

Proposition 5. Let $\mathcal{H}, \mathcal{H}^{\prime}$ be two different Hamiltonians associated to an almost-regular Lagrangian $L$. Let $H, H^{\prime}$ be the corresponding Hamiltonian forms (8). Their pull-backs $i_{N}^{*} H$ and $i_{N}^{*} H^{\prime}$ onto the Lagrangian constraint manifold $N_{L}$ coincide with each other.

It follows that, if an almost-regular Lagrangian admits associated Hamiltonians $\mathcal{H}$, it defines a unique constrained Hamiltonian form $H_{N}=i_{N}^{*} H$ on the Lagrangian constraint manifold $N_{L}$ and a unique constrained Lagrangian $L_{N}=h_{0}\left(H_{N}\right)$ (11) on the jet manifold $J^{1} N_{L}$ of the fiber bundle $N_{L} \rightarrow X$. For any Hamiltonian $\mathcal{H}$ associated to $L$, every solution $r$ of the Hamilton equations which lives in the Lagrangian constraint space $N_{L}$ is a solution of the constrained Hamilton equations for $L_{N}$.

Theorem 6. Let an almost-regular Lagrangian $L$ admit associated Hamiltonians. A section $\bar{s}$ of the jet bundle $J^{1} Y \rightarrow X$ is a solution of the Cartan equations for $L$ iff $\widehat{L} \circ \bar{s}$ is a solution of the constrained Hamilton equations. In particular, any solution $r$ of the constrained Hamilton equations provides the solution $\bar{s}=\widehat{H} \circ r$ of the Cartan equations.

Theorem 6 shows that the constrained Hamilton equations and the Cartan equations are quasi-equivalent. Thus, one can associate to an almost-regular Lagrangian $L$ (4) a unique constrained Lagrangian system on the constraint Lagrangian manifold $N_{L}(15)$. Let us compare quantizations of these Lagrangian systems on $Y$ and $N_{L} \subset \Pi$ in the case of an almost-regular quadratic Lagrangian $L$. 


\section{QUADRATIC DEGENERATE SYSTEMS}

Given a fiber bundle $Y \rightarrow X$, let us consider a quadratic Lagrangian $L$ (3), where $a, b$ and $c$ are local functions on $Y$. This property is coordinate-independent since $J^{1} Y \rightarrow Y$ is an affine bundle modelled over the vector bundle $T^{*} X \underset{Y}{\otimes} V Y$, where $V Y$ denotes the vertical tangent bundle of $Y \rightarrow X$. The associated Legendre map (13) reads

$$
p_{i}^{\lambda} \circ \widehat{L}=a_{i j}^{\lambda \mu} y_{\mu}^{j}+b_{i}^{\lambda} .
$$

Let a Lagrangian $L(3)$ be almost-regular, i.e., the matrix function $a$ is a linear bundle morphism

$$
a: T^{*} X \underset{Y}{\otimes} V Y \rightarrow \Pi, \quad p_{i}^{\lambda}=a_{i j}^{\lambda \mu} \bar{y}_{\mu}^{j},
$$

of constant rank, where $\left(x^{\lambda}, y^{i}, \bar{y}_{\lambda}^{i}\right)$ are bundle coordinates on $T^{*} X \underset{Y}{\otimes} V Y$. Then the Lagrangian constraint space $N_{L}$ (17) is an affine subbundle of the Legendre bundle $\Pi \rightarrow Y$. Hence, $N_{L} \rightarrow Y$ has a global section. For the sake of simplicity, let us assume that it is the canonical zero section $\widehat{0}(Y)$ of $\Pi \rightarrow Y$. The kernel of the Legendre map (17) is also an affine subbundle of the affine jet bundle $J^{1} Y \rightarrow Y$. Therefore, it admits a global section

$$
\Gamma: Y \rightarrow \operatorname{Ker} \widehat{L} \subset J^{1} Y, \quad a_{i j}^{\lambda \mu} \Gamma_{\mu}^{j}+b_{i}^{\lambda}=0,
$$

which is a connection on $Y \rightarrow X$. If the Lagrangian (3) is regular, the connection (19) is unique.

The forthcoming theorems are the key points of our analysis of quadratic degenerate systems (Giachetta et al., 1997, 1999).

Theorem \%. There exists a linear bundle morphism

$$
\sigma: \Pi \underset{Y}{\rightarrow} T^{*} X \underset{Y}{\otimes} V Y, \quad \bar{y}_{\lambda}^{i} \circ \sigma=\sigma_{\lambda \mu}^{i j} p_{j}^{\mu},
$$

such that

$$
a \circ \sigma \circ a=a, \quad a_{i j}^{\lambda \mu} \sigma_{\mu \alpha}^{j k} a_{k b}^{\alpha \nu}=a_{i b}^{\lambda \nu}
$$

Note that $\sigma$ is not unique, but it falls into the sum $\sigma=\sigma_{0}+\sigma_{1}$ such that

$$
\sigma_{0} \circ a \circ \sigma_{0}=\sigma_{0}, \quad a \circ \sigma_{1}=\sigma_{1} \circ a=0,
$$

where $\sigma_{0}$ is uniquely defined. For instance, there exists a nondegenerate map $\sigma(20)$. 
Theorem 8. There are the splittings

$$
\begin{aligned}
& J^{1} Y=\mathcal{S}\left(J^{1} Y\right) \underset{Y}{\oplus} \mathcal{F}\left(J^{1} Y\right)=\operatorname{Ker} \widehat{L} \underset{Y}{\oplus} \operatorname{Im}\left(\sigma_{0} \circ \widehat{L}\right), \\
& y_{\lambda}^{i}=\mathcal{S}_{\lambda}^{i}+\mathcal{F}_{\lambda}^{i}=\left[y_{\lambda}^{i}-\sigma_{0 \lambda \alpha}^{i k}\left(a_{k j}^{\alpha \mu} y_{\mu}^{j}+b_{k}^{\alpha}\right)\right]+\left[\sigma_{0 \lambda \alpha}^{i k}\left(a_{k j}^{\alpha \mu} y_{\mu}^{j}+b_{k}^{\alpha}\right)\right], \\
& \Pi=\mathcal{R}(\Pi) \underset{Y}{\oplus} \mathcal{P}(\Pi)=\operatorname{Ker} \sigma_{0} \underset{Y}{\oplus} N_{L}, \\
& p_{i}^{\lambda}=\mathcal{R}_{i}^{\lambda}+\mathcal{P}_{i}^{\lambda}=\left[p_{i}^{\lambda}-a_{i j}^{\lambda \mu} \sigma_{0 \mu \alpha}^{j k} p_{k}^{\alpha}\right]+\left[a_{i j}^{\lambda \mu} \sigma_{0 \mu \alpha}^{j k} p_{k}^{\alpha}\right] .
\end{aligned}
$$

The relations (22) lead to the equalities

$$
a_{i j}^{\lambda \mu} \mathcal{S}_{\mu}^{j}=0, \quad \sigma_{0 \mu \alpha}^{j k} \mathcal{R}_{k}^{\alpha}=0, \quad \sigma_{1 \mu \alpha}^{j k} \mathcal{P}_{k}^{\alpha}=0, \quad \mathcal{R}_{i}^{\lambda} \mathcal{F}_{\lambda}^{i}=0 .
$$

By virtue of these equalities, the Lagrangian (3) takes the form

$$
L=\mathcal{L} \omega, \quad \mathcal{L}=\frac{1}{2} a_{i j}^{\lambda \mu} \mathcal{F}_{\lambda}^{i} \mathcal{F}_{\mu}^{j}+c^{\prime} .
$$

One can show that, this Lagrangian admits a set of associated Hamiltonians

$$
\mathcal{H}_{\Gamma}=\left(\mathcal{R}_{i}^{\lambda}+\mathcal{P}_{i}^{\lambda}\right) \Gamma_{\lambda}^{i}+\frac{1}{2} \sigma_{0 \lambda \mu}^{i j} \mathcal{P}_{i}^{\lambda} \mathcal{P}_{j}^{\mu}+\frac{1}{2} \sigma_{1 \lambda \mu}^{i j} \mathcal{R}_{i}^{\lambda} \mathcal{R}_{j}^{\mu}-c^{\prime}
$$

indexed by connections $\Gamma$ (19). Accordingly, the Lagrangian constraint manifold (17) is given by the reducible constraints

$$
\mathcal{R}_{i}^{\lambda}=p_{i}^{\lambda}-a_{i j}^{\lambda \mu} \sigma_{0}^{j k} p_{k}^{\alpha}=0 .
$$

Given a Hamiltonian $\mathcal{H}_{\Gamma}$, the corresponding Lagrangian (10) reads

$$
\mathcal{L}_{\mathcal{H}_{\Gamma}}=\mathcal{R}_{i}^{\lambda}\left(\mathcal{S}_{\lambda}^{i}-\Gamma_{\lambda}^{i}\right)+\mathcal{P}_{i}^{\lambda} \mathcal{F}_{\lambda}^{i}-\frac{1}{2} \sigma_{0 \lambda \mu}^{i j} \mathcal{P}_{i}^{\lambda} \mathcal{P}_{j}^{\mu}-\frac{1}{2} \sigma_{1 \lambda \mu}^{i j} \mathcal{R}_{i}^{\lambda} \mathcal{R}_{j}^{\mu}+c^{\prime}
$$

Its restriction (11) to the Lagrangian constraint manifold $N_{L}(28)$ is

$$
L_{N}=\mathcal{L}_{N} \omega, \quad \mathcal{L}_{N}=\mathcal{P}_{i}^{\lambda} \mathcal{F}_{\lambda}^{i}-\frac{1}{2} \sigma_{0}{ }_{\lambda \mu}^{i j} \mathcal{P}_{i}^{\lambda} \mathcal{P}_{j}^{\mu}+c^{\prime}
$$

It is independent of the choice of a Hamiltonian (27). Note that the Lagrangian $\mathcal{L}_{N}$ may admit gauge symmetries due to the term $\mathcal{P}_{i}^{\lambda} \mathcal{F}_{\lambda}^{i}$.

The Hamiltonian $\mathcal{H}_{\Gamma}$ yields the Hamiltonian map $\widehat{H}_{\Gamma}$ (14) and the projector

$$
T=\widehat{L} \circ \widehat{H}_{\Gamma}, \quad p_{i}^{\lambda} \circ T=T_{i \mu}^{\lambda j} p_{j}^{\mu}=a_{i k}^{\lambda \nu} \sigma_{0 \nu \mu}^{k j} p_{j}^{\mu}=\mathcal{P}_{i}^{\lambda},
$$


from $\Pi$ onto its summand $N_{L}$ in the decomposition (24). It obeys the relations

$$
\sigma \circ T=\sigma_{0}, \quad T \circ a=a .
$$

The projector $T$ (31) is a linear morphism over $\operatorname{Id} Y$. Therefore, $T: \Pi \rightarrow N_{L}$ is a vector bundle. Let us consider the pull-back $L_{\Pi}=T^{*} L_{N}$ of the constrained Lagrangian $L_{N}$ (30) onto П. By virtue of the relations (25), it is given by the coordinate expression

$$
L_{\Pi}=\mathcal{L}_{\Pi} \omega, \quad \mathcal{L}_{\Pi}=p_{i}^{\lambda} \mathcal{F}_{\lambda}^{i}-\frac{1}{2} \sigma_{0 \lambda \mu}^{i j} p_{i}^{\lambda} p_{j}^{\mu}+c^{\prime}
$$

This Lagrangian is gauge-invariant under the subgroup of the gauge group of vertical automorphisms $\Phi$ of the affine bundle $\Pi \rightarrow Y$ such that $T \circ \Phi=T$. Clearly, this subgroup coincides with the gauge group Aut Ker $\sigma_{0}$ of vertical automorphisms of the vector bundle Ker $\sigma_{0} \rightarrow Y$.

In fact, the splittings (23) and (24) result from the splitting of the vector bundle

$$
T^{*} X \underset{Y}{\otimes} V Y=\operatorname{Ker} a \underset{Y}{\oplus} E
$$

which can be provided with the adapted coordinates $\left(\bar{y}^{a}, \bar{y}^{A}\right)$ such that $a(18)$ is brought into a diagonal matrix with nonvanishing components $a_{A A}$. Then the Legendre bundle $\Pi \rightarrow Y(6)$ is endowed with the dual (nonholonomic) coordinates $\left(p_{a}, p_{A}\right)$ where $p_{A}$ are coordinates on the Lagrangian constraint manifold $N_{L}$, given by the irreducible constraints $p_{a}=0$. Written relative to these coordinates, $\sigma_{0}$ becomes the diagonal matrix

$$
\sigma_{0}^{A A}=\left(a_{A A}\right)^{-1}, \quad \sigma_{0}^{a a}=0,
$$

while $\sigma_{1}^{A a}=\sigma_{1}^{A B}=0$. Moreover, one can choose the coordinates $\bar{y}^{a}$ (accordingly, $p_{a}$ ) and the map $\sigma$ (20) such that $\sigma_{1}$ becomes a diagonal matrix with nonvanishing positive components $\sigma_{1}^{a a}=\mathcal{V}^{-1}$, where $\mathcal{V} \omega$ is a volume form on $X$. We further follow this choice of the adapted coordinates $\left(p_{a}, p_{A}\right)$. Let us write

$$
p_{a}=M_{a \lambda}^{i} p_{i}^{\lambda}, \quad p_{A}=M_{A \lambda}^{i} p_{i}^{\lambda},
$$

where $M$ are the matrix functions on $Y$ obeying the relations

$$
M_{a \lambda}^{i} a_{i j}^{\lambda \mu}=0, \quad M_{i}^{-1 \lambda a} \sigma_{0 \lambda}^{i}=0, \quad M_{a \lambda}^{i} \mathcal{P}_{i}^{\lambda}=0, \quad M_{A \lambda}^{i} \mathcal{R}_{i}^{\lambda}=0 .
$$

Then the Lagrangian $L_{N}(30)$ with respect to the adapted coordinates $\left(p_{a}, p_{A}\right)$ takes the form

$$
\mathcal{L}_{N}=M_{i}^{-1 \lambda A} p_{A} \mathcal{F}_{\lambda}^{i}-\frac{1}{2} \sum_{A}\left(a_{A A}\right)^{-1}\left(p_{A}\right)^{2}+c^{\prime},
$$




\section{QUANTIZATION}

Let us quantize a Lagrangian system with the Lagrangian $L_{N}(30)$ on the constraint manifold $N_{L}$ (28). In the framework of a perturbative quantum field theory, we should assume that $X=\mathbb{R}^{n}$ and $Y \rightarrow X$ is a trivial affine bundle. It follows that both the original coordinates $\left(x^{\lambda}, y^{i}, p_{i}^{\lambda}\right)$ and the adapted coordinates $\left(x^{\lambda}, y^{i}, p_{a}, p_{A}\right)$ on the Legendre bundle $\Pi$ are global. Passing to field theory on an Euclidean space $\mathbb{R}^{n}$, we also assume that the matrix $a$ in the Lagrangian $L(26)$ is positive-definite, i.e., $a_{A A}>0$.

Let us start from a Lagrangian (30) without gauge symmetries. Since the Lagrangian constraint space $N_{L}$ can be equipped with the adapted coordinates $p_{A}$, the generating functional of Euclidean Green functions of the Lagrangian system in question reads

$$
Z=\mathcal{N}^{-1} \int \exp \left\{\int\left(\mathcal{L}_{N}+\frac{1}{2} \operatorname{tr} \ln \bar{\sigma}_{0}+i J_{i} y^{i}+i J^{A} p_{A}\right) \omega\right\} \prod_{x}\left[d p_{A}(x)\right][d y(x)],
$$

where $\mathcal{L}_{N}$ is given by the expression (36) and $\bar{\sigma}_{0}$ is the square matrix

$$
\bar{\sigma}_{0}^{A B}=M_{i}^{-1 \lambda A} M_{j}^{-1 \mu B} \sigma_{0 \lambda \mu}^{i j}=\delta^{A B}\left(a_{A A}\right)^{-1}
$$

The generating functional (37) a Gaussian integral of variables $p_{A}(x)$. Its integration with respect to $p_{A}(x)$ under the condition $J^{A}=0$ restarts the generating functional

$$
Z=\mathcal{N}^{-1} \int \exp \left\{\int\left(\mathcal{L}+i J_{i} y^{i}\right) \omega\right\} \prod_{x}[d y(x)]
$$

of the original Lagrangian field system on $Y$ with the Lagrangian (26). However, the generating functional (37) can not be rewritten with respect to the original variables $p_{i}^{\mu}$, unless $a$ is a nondegenerate matrix function.

In order to overcome this difficulty, let us consider a Lagrangian system on the whole Legendre manifold $\Pi$ with the Lagrangian $L_{\Pi}$ (33). Since this Lagrangian is constant along the fibers of the vector bundle $\Pi \rightarrow N_{L}$, an integration of the generating functional of this field model with respect to variables $p_{a}(x)$ should be finite. One can choose the generating functional in the form

$$
Z=\mathcal{N}^{-1} \int \exp \left\{\int\left(\mathcal{L}_{\Pi}-\frac{1}{2} \sigma_{1}^{i j} p_{i}^{\lambda} p_{j}^{\mu}+\frac{1}{2} \operatorname{tr} \ln \sigma+i J_{i} y^{i}+i J_{\mu}^{i} p_{i}^{\mu}\right) \omega\right\} \prod_{x}[d p(x)][d y(x)] .
$$

Its integration with respect to momenta $p_{i}^{\lambda}(x)$ restarts the generating functional (38) of the original Lagrangian system on $Y$. In order to obtain the generating functional (39), one can follow a procedure of quantization of gauge-invariant Lagrangian systems. In the case of the 
Lagrangian $L_{\Pi}(33)$, this procedure is rather trivial, since the space of momenta variables $p_{a}(x)$ coincides with the translation subgroup of the gauge group Aut $\operatorname{Ker} \sigma_{0}$.

Now let us suppose that the Lagrangian $L_{N}(30)$ and, consequently, the Lagrangian $L_{\Pi}$ (33) are invariant under some gauge group $G_{X}$ of vertical automorphisms of the fiber bundle $Y \rightarrow X$ (and the induced automorphisms of $\Pi \rightarrow X$ ) which acts freely on the space of sections of $Y \rightarrow X$. Its infinitesimal generators are represented by vertical vector fields $u=u^{i}\left(x^{\mu}, y^{j}\right) \partial_{i}$ on $Y \rightarrow X$ which give rise to the vector fields

$$
\bar{u}=u^{i} \partial_{i}-\partial_{j} u^{i} p_{i}^{\lambda} \partial_{\lambda}^{j}+d_{\lambda} u^{i} \partial_{i}^{\lambda}, \quad d_{\lambda}=\partial_{\lambda}+y_{\lambda}^{i} \partial_{i}
$$

on $\Pi \underset{Y}{\times} J^{1} Y$. Let us also assume that $G_{X}$ is indexed by $m$ parameter functions $\xi^{r}(x)$ such that $u=u^{i}\left(x^{\lambda}, y^{j}, \xi^{r}\right) \partial_{i}$, where

$$
u^{i}\left(x^{\lambda}, y^{j}, \xi^{r}\right)=u_{r}^{i}\left(x^{\lambda}, y^{j}\right) \xi^{r}+u_{r}^{i \mu}\left(x^{\lambda}, y^{j}\right) \partial_{\mu} \xi^{r}
$$

are linear first order differential operators on the space of parameters $\xi^{r}(x)$. The vector fields $u\left(\xi^{r}\right)$ must satisfy the commutation relations

$$
\left[u\left(\xi^{q}\right), u\left(\xi^{\prime p}\right)\right]=u\left(c_{p q}^{r} \xi^{\prime p} \xi^{q}\right)
$$

where $c_{p q}^{r}$ are structure constants. The Lagrangian $L_{\Pi}(33)$ is invariant under the above mentioned gauge transformations iff its Lie derivative $\mathbf{L}_{\bar{u}} L_{\Pi}$ along vector fields (40) vanishes, i.e.,

$$
\left(u^{i} \partial_{i}-\partial_{j} u^{i} p_{i}^{\lambda} \partial_{\lambda}^{j}+d_{\lambda} u^{i} \partial_{i}^{\lambda}\right) \mathcal{L}_{\Pi}=0
$$

Since the operator $\mathbf{L}_{\bar{u}}$ is linear in momenta $p_{i}^{\mu}$, the condition (42) falls into the independent conditions

$$
\begin{aligned}
& \left(u^{k} \partial_{k}-\partial_{j} u^{k} p_{k}^{\nu} \partial_{\nu}^{j}+d_{\nu} u^{j} \partial_{j}^{\nu}\right)\left(p_{i}^{\lambda} \mathcal{F}_{\lambda}^{i}\right)=0, \\
& \left(u^{k} \partial_{k}-\partial_{j} u^{k} p_{k}^{\nu} \partial_{\nu}^{j}\right)\left(\sigma_{0 \lambda \mu}^{i j} p_{i}^{\lambda} p_{j}^{\mu}\right)=0 \\
& u^{i} \partial_{i} c^{\prime}=0 .
\end{aligned}
$$

It follows that the Lagrangian $L_{\Pi}$ is gauge-invariant iff its three summands are separately gauge-invariant.

Note that, if the Lagrangian $L_{\Pi}$ on $\Pi$ is gauge-invariant, the original Lagrangian $L(26)$ is also invariant under the same gauge transformations. Indeed, one obtains at once from the condition (43) that

$$
\bar{u}\left(\mathcal{F}_{\mu}^{i}\right)=\partial_{j} u^{i} \mathcal{F}_{\mu}^{j},
$$


i.e., the quantity $\mathcal{F}$ is transformed as the dual of momenta $p$. Then the condition (44) shows that the quantity $\sigma_{0} p$ is transformed by the same law as $\mathcal{F}$. It follows that the term $a \mathcal{F} \mathcal{F}$ in the Lagrangian $L(26)$ is transformed exactly as $a\left(\sigma_{0} p\right)\left(\sigma_{0} p\right)=\sigma_{0} p p$, i.e., is gauge-invariant. Then this Lagrangian is gauge-invariant due to the equality (45).

Since $\mathcal{S}_{\lambda}^{i}=y_{\lambda}^{i}-\mathcal{F}_{\lambda}^{i}$, one can easily derive from the formula (46) the transformation law

$$
\bar{u}\left(\mathcal{S}_{\mu}^{i}\right)=d_{\mu} u^{i}-\partial_{j} u^{i} \mathcal{F}_{\mu}^{j}=d_{\mu} u^{i}-\partial_{j} u^{i}\left(y_{\mu}^{j}-\mathcal{S}_{\mu}^{j}\right)=\partial_{\mu} u^{i}+\partial_{j} u^{i} \mathcal{S}_{\mu}^{j}
$$

of $\mathcal{S}$. A glance at this expression shows that the gauge group $G_{X}$ acts freely on the space of sections $\mathcal{S}(x)$ of the fiber bundle $\operatorname{Ker} \widehat{L} \rightarrow Y$ in the splitting (23). Let the number $m$ of parameters of the gauge group $G_{X}$ do not exceed the fiber dimension of $\operatorname{Ker} \widehat{L} \rightarrow Y$. Then some combinations $b_{i}^{r \mu} \mathcal{S}_{\mu}^{i}$ of $\mathcal{S}_{\mu}^{i}$ can be used as the gauge condition

$$
b_{i}^{r \mu} \mathcal{S}_{\mu}^{i}(x)-\alpha^{r}(x)=0
$$

similar to the generalized Lorentz gauge in Yang-Mills gauge theory.

Turn now to quantization of a Lagrangian system with the gauge-invariant Lagrangian $L_{\Pi}$ (33). In accordance with the well-known quantization procedure, let us modify the generating functional (39) as follows

$$
\begin{aligned}
Z= & \mathcal{N}^{-1} \int \exp \left\{\int\left(\mathcal{L}_{\Pi}-\frac{1}{2} \sigma_{1}{ }_{\lambda \mu}^{i j} p_{i}^{\lambda} p_{j}^{\mu}+\frac{1}{2} \operatorname{tr} \ln \sigma-\frac{1}{2} h_{r s} \alpha^{r} \alpha^{s}+i J_{i} y^{i}+i J_{\mu}^{i} p_{i}^{\mu}\right) \omega\right\} \\
& \Delta \prod_{x} \times{ }^{r} \delta\left(b_{i}^{r \mu} \mathcal{S}_{\mu}^{i}(x)-\alpha^{r}(x)\right)[d \alpha(x)][d p(x)][d y(x)]= \\
\mathcal{N}^{\prime-1} \int & \exp \left\{\int\left(\mathcal{L}_{\Pi}-\frac{1}{2} \sigma_{1}{ }_{1 j \mu}^{i j} p_{i}^{\lambda} p_{j}^{\mu}+\frac{1}{2} \operatorname{tr} \ln \sigma-\frac{1}{2} h_{r s} b_{i}^{r \mu} b_{j}^{s \lambda} \mathcal{S}_{\mu}^{i} \mathcal{S}_{\lambda}^{j}+i J_{i} y^{i}+i J_{\mu}^{i} p_{i}^{\mu}\right) \omega\right\} \\
& \Delta \prod_{x}[d p(x)][d y(x)],
\end{aligned}
$$

where

$$
\int \exp \left\{\int\left(-\frac{1}{2} h_{r s} \alpha^{r} \alpha^{s}\right) \omega\right\} \prod_{x}[d \alpha(x)]
$$

is a Gaussian integral, and the factor $\Delta$ is defined by the condition

$$
\Delta \int \prod_{x} \stackrel{r}{\times} \delta\left(u(\xi)\left(b_{i}^{r \mu} \mathcal{S}_{\mu}^{i}\right)\right)[d \xi(x)]=1 .
$$

We have the linear second order differential operator

$$
M_{s}^{r} \xi^{s}=u(\xi)\left(b_{i}^{r \mu} \mathcal{S}_{\mu}^{i}(x)\right)=b_{i}^{r \mu}\left(\partial_{\mu} u^{i}(\xi)+\partial_{j} u^{i}(\xi) \mathcal{S}_{\mu}^{j}\right)
$$


on the parameter functions $\xi(x)$, and obtain $\Delta=\operatorname{det} M$. Then the generating functional (48) takes the form

$$
\begin{aligned}
Z= & \mathcal{N}^{\prime-1} \int \exp \left\{\int \left(\mathcal{L}_{\Pi}-\frac{1}{2} \sigma_{1 \lambda \mu}^{i j} p_{i}^{\lambda} p_{j}^{\mu}+\frac{1}{2} \operatorname{tr} \ln \sigma-\frac{1}{2} h_{r s} b_{i}^{r \mu} b_{j}^{s \lambda} \mathcal{S}_{\mu}^{i} \mathcal{S}_{\lambda}^{j}-\bar{c}_{r} M_{s}^{r} c^{s}+\right.\right. \\
& \left.\left.i J_{i} y^{i}+i J_{\mu}^{i} p_{i}^{\mu}\right) \omega\right\} \prod_{x}[d \bar{c}][d c][d p(x)][d y(x)],
\end{aligned}
$$

where $\bar{c}_{r}, c^{s}$ are odd ghost fields. Integrating $Z$ (50) with respect to momenta under the condition $J_{\mu}^{i}=0$, we come to the generating functional

$$
Z=\mathcal{N}^{\prime-1} \int \exp \left\{\int\left(\mathcal{L}-\frac{1}{2} h_{r s} b_{i}^{r \mu} b_{j}^{s \lambda} \mathcal{S}_{\mu}^{i} \mathcal{S}_{\lambda}^{j}-\bar{c}_{r} M_{s}^{r} c^{s}+i J_{i} y^{i}\right) \omega\right\} \prod_{x}[d \bar{c}][d c][d y(x)]
$$

of the original field model on $Y$ with the gauge-invariant Lagrangian $L$ (26).

Note that the Lagrangian

$$
\mathcal{L}^{\prime}=\mathcal{L}-\frac{1}{2} h_{r s} b_{i}^{r \mu} b_{j}^{s \lambda} \mathcal{S}_{\mu}^{i} \mathcal{S}_{\lambda}^{j}-\bar{c}_{r} M_{s}^{r} c^{s}
$$

fails to be gauge-invariant, but it admits the BRST symmetry whose odd operator reads

$$
\begin{aligned}
\vartheta= & u^{i}\left(x^{\mu}, y^{i}, c^{s}\right) \partial_{i}+d_{\lambda} u^{i}\left(x^{\mu}, y^{i}, c^{s}\right) \partial_{i}^{\lambda}+\bar{v}_{r}\left(x^{\mu}, y^{i}, y_{\mu}^{i}\right) \frac{\partial}{\partial \bar{c}_{r}}+ \\
& v^{r}\left(x^{\mu}, y^{i}, c^{s}\right) \frac{\partial}{\partial c^{r}}+d_{\lambda} v^{r}\left(x^{\mu}, y^{i}, c^{s}\right) \frac{\partial}{\partial c_{\lambda}^{r}}+d_{\mu} d_{\lambda} v^{r}\left(x^{\mu}, y^{i}, c^{s}\right) \frac{\partial}{\partial c_{\mu \lambda}^{r}} \\
d_{\lambda}= & \partial_{\lambda}+y_{\lambda}^{i} \partial_{i}+y_{\lambda \mu}^{i} \partial_{i}^{\mu}+c_{\lambda}^{r} \frac{\partial}{\partial c^{r}}+c_{\lambda \mu}^{r} \frac{\partial}{\partial c_{\mu}^{r}}
\end{aligned}
$$

Its components $u^{i}\left(x^{\mu}, y^{i}, c^{s}\right)$ are given by the expression (41) where parameter functions $\xi^{r}(x)$ are replaced with the ghosts $c^{r}$. The components $\bar{v}_{r}$ and $v^{r}$ of the BRST operator $\vartheta$ can be derived from the condition

$$
\vartheta\left(\mathcal{L}^{\prime}\right)=-h_{r s} M_{q}^{r} b_{j}^{s \lambda} \mathcal{S}_{\lambda}^{j} c^{q}-\bar{v}_{r} M_{q}^{r} c^{q}+\bar{c}_{r} \vartheta\left(\vartheta\left(b_{j}^{r \lambda} \mathcal{S}_{\lambda}^{j}\right)\right)=0
$$

of the BRST invariance of $\mathcal{L}^{\prime}$. This condition falls into the two independent relations

$$
\begin{aligned}
& h_{r s} M_{q}^{r} b_{j}^{s \lambda} \mathcal{S}_{\lambda}^{j}+\bar{v}_{r} M_{q}^{r}=0, \\
& \vartheta\left(c^{q}\right)\left(\vartheta\left(c^{p}\right)\left(b^{r \lambda} \mathcal{S}_{\lambda}^{j}\right)\right)=u\left(c^{p}\right)\left(u\left(c^{q}\right)\left(b_{j}^{r \lambda} \mathcal{S}_{\lambda}^{j}\right)\right)+u\left(v^{r}\right)\left(b_{j}^{r \lambda} \mathcal{S}_{\lambda}^{j}\right)= \\
& u\left(\frac{1}{2} c_{p q}^{r} c^{p} c^{q}+v^{r}\right)\left(b_{j}^{r \lambda} \mathcal{S}_{\lambda}^{j}\right)=0 .
\end{aligned}
$$

Hence, we obtain

$$
\bar{v}_{r}=-h_{r s} b_{j}^{s \lambda} \mathcal{S}_{\lambda}^{j}, \quad v^{r}=-\frac{1}{2} c_{p q}^{r} c^{p} c^{q}
$$




\section{HAMILTONIAN GAUGE THEORY}

For example, let us consider gauge theory of principal connections on a principal bundle $P \rightarrow X$ with a structure Lie group $G$. Principal connections on $P \rightarrow X$ are represented by sections of the affine bundle

$$
C=J^{1} P / G \rightarrow X
$$

modelled over the vector bundle $T^{*} X \otimes V_{G} P[5]$. Here, $V_{G} P=V P / G$ is the fiber bundle in Lie algebras $\mathfrak{g}$ of the group $G$. Given the basis $\left\{\varepsilon_{r}\right\}$ for $\mathfrak{g}$, we obtain the local fiber bases $\left\{e_{r}\right\}$ for $V_{G} P$. The connection bundle $C(55)$ is coordinated by $\left(x^{\mu}, a_{\mu}^{r}\right)$ such that, written relative to these coordinates, sections $A=A_{\mu}^{r} d x^{\mu} \otimes e_{r}$ of $C \rightarrow X$ are the familiar local connection one-forms, regarded as gauge potentials.

There is one-to-one correspondence between the sections $\xi=\xi^{r} e_{r}$ of $V_{G} P \rightarrow X$ and the vector fields on $P$ which are infinitesimal generators of one-parameter groups of vertical automorphisms (gauge transformations) of $P$. Any section $\xi$ of $V_{G} P \rightarrow X$ yields the vector field

$$
u(\xi)=u_{\mu}^{r} \frac{\partial}{\partial a_{\mu}^{r}}=\left(c_{p q}^{r} a_{\mu}^{p} \xi^{q}+\partial_{\mu} \xi^{r}\right) \frac{\partial}{\partial a_{\mu}^{r}}
$$

on $C$, where $c_{p q}^{r}$ are the structure constants of the Lie algebra $\mathfrak{g}$.

The configuration space of gauge theory is the jet manifold $J^{1} C$ equipped with the coordinates $\left(x^{\lambda}, a_{\lambda}^{m}, a_{\mu \lambda}^{m}\right)$. It admits the canonical splitting (23) given by the coordinate expression

$$
a_{\mu \lambda}^{r}=\mathcal{S}_{\mu \lambda}^{r}+\mathcal{F}_{\mu \lambda}^{r}=\frac{1}{2}\left(a_{\mu \lambda}^{r}+a_{\lambda \mu}^{r}-c_{p q}^{r} a_{\mu}^{p} a_{\lambda}^{q}\right)+\frac{1}{2}\left(a_{\mu \lambda}^{r}-a_{\lambda \mu}^{r}+c_{p q}^{r} a_{\mu}^{p} a_{\lambda}^{q}\right),
$$

where $\mathcal{F}$ is the strength of gauge fields up to the factor $1 / 2$. The Yang-Mills Lagrangian on the configuration space $J^{1} C$ reads

$$
L_{\mathrm{YM}}=a_{p q}^{G} g^{\lambda \mu} g^{\beta \nu} \mathcal{F}_{\lambda \beta}^{p} \mathcal{F}_{\mu \nu}^{q} \sqrt{|g|} \omega, \quad g=\operatorname{det}\left(g_{\mu \nu}\right),
$$

where $a^{G}$ is a non-degenerate $G$-invariant metric in the dual of the Lie algebra of $\mathfrak{g}$ and $g$ is a pseudo-Riemannian metric on $X$.

The phase space $\Pi(6)$ of the gauge theory is endowed with the canonical coordinates $\left(x^{\lambda}, a_{\lambda}^{p}, p_{q}^{\mu \lambda}\right)$. It admits the canonical splitting (24) given by the coordinate expression

$$
p_{m}^{\mu \lambda}=\mathcal{R}_{m}^{\mu \lambda}+\mathcal{P}_{m}^{\mu \lambda}=p_{m}^{(\mu \lambda)}+p_{m}^{[\mu \lambda]}=\frac{1}{2}\left(p_{m}^{\mu \lambda}+p_{m}^{\lambda \mu}\right)+\frac{1}{2}\left(p_{m}^{\mu \lambda}-p_{m}^{\lambda \mu}\right) .
$$


With respect to this splitting, the Legendre map induced by the Lagrangian (58) takes the form

$$
\begin{aligned}
p_{m}^{(\mu \lambda)} \circ \widehat{L}_{Y M} & =0, \\
p_{m}^{[\mu \lambda]} \circ \widehat{L}_{Y M} & =4 a_{m n}^{G} g^{\mu \alpha} g^{\lambda \beta} \mathcal{F}_{\alpha \beta}^{n} \sqrt{|g|} .
\end{aligned}
$$

The equalities (60) define the Lagrangian constraint space $N_{L}$ of Hamiltonian gauge theory. Obviously, it is an imbedded submanifold of $\Pi$, and the Lagrangian $L_{\mathrm{YM}}$ is almost-regular.

In order to construct an associated Hamiltonian, let us consider a connection $\Gamma(19)$ on the fiber bundle $C \rightarrow X$ which take their values into $\operatorname{Ker} \widehat{L}$, i.e.,

$$
\Gamma_{\lambda \mu}^{r}-\Gamma_{\mu \lambda}^{r}+c_{p q}^{r} a_{\lambda}^{p} a_{\mu}^{q}=0
$$

Given a symmetric linear connection $K$ on $X$ and a principal connection $B$ on $P \rightarrow X$, this connection reads

$$
\Gamma_{\lambda \mu}^{r}=\frac{1}{2}\left[\partial_{\mu} B_{\lambda}^{r}+\partial_{\lambda} B_{\mu}^{r}-c_{p q}^{r} a_{\lambda}^{p} a_{\mu}^{q}+c_{p q}^{r}\left(a_{\lambda}^{p} B_{\mu}^{q}+a_{\mu}^{p} B_{\lambda}^{q}\right)\right]-K_{\lambda}{ }^{\beta}{ }_{\mu}\left(a_{\beta}^{r}-B_{\beta}^{r}\right) .
$$

The corresponding Hamiltonian (27) associated to $L_{\mathrm{YM}}$ is

$$
\mathcal{H}_{\Gamma}=p_{r}^{\lambda \mu} \Gamma_{\lambda \mu}^{r}+a_{G}^{m n} g_{\mu \nu} g_{\lambda \beta} p_{m}^{[\mu \lambda]} p_{n}^{[\nu \beta]} \sqrt{|g|}
$$

Then we obtain the Lagrangian

$$
\mathcal{L}_{N}=p_{r}^{[\lambda \mu]} \mathcal{F}_{\lambda \mu}^{r}-a_{G}^{m n} g_{\mu \nu} g_{\lambda \beta} p_{m}^{[\mu \lambda]} p_{n}^{[\nu \beta]} \sqrt{|g|}
$$

(30) on the Lagrangian constraint manifold (60) and its pull-back

$$
L_{\Pi}=\mathcal{L}_{\Pi} \omega, \quad \mathcal{L}_{\Pi}=p_{r}^{\lambda \mu} \mathcal{F}_{\lambda \mu}^{r}-a_{G}^{m n} g_{\mu \nu} g_{\lambda \beta} p_{m}^{[\mu \lambda]} p_{n}^{[\nu \beta]} \sqrt{|g|}
$$

(33) onto П.

Both the Lagrangian $L_{\mathrm{YM}}(58)$ on $C$ and the Lagrangian $L_{\Pi}(62)$ on $\Pi$ are invariant under gauge transformations whose infinitesimal generators are the lifts

$$
\begin{aligned}
& J^{1} u(\xi)=\left(c_{p q}^{r} a_{\mu}^{p} \xi^{q}+\partial_{\mu} \xi^{r}\right) \frac{\partial}{\partial a_{\mu}^{r}}+\left(c_{p q}^{r}\left(a_{\lambda \mu}^{p} \xi^{q}+a_{\mu}^{p} \partial_{\lambda} \xi^{q}\right)+\partial_{\lambda} \partial_{\mu} \xi^{r}\right) \frac{\partial}{\partial a_{\lambda \mu}^{r}} \\
& \bar{u}(\xi)=J^{1} u(\xi)-c_{p q}^{r} p_{r}^{\lambda \mu} \xi^{q} \frac{\partial}{\partial p_{p}^{\lambda \mu}}
\end{aligned}
$$


of the vector fields (56) onto $J^{1} C$ and $\Pi \underset{C}{\times} J^{1} C$, respectively. We have the transformation laws

$$
J^{1} u(\xi)\left(\mathcal{F}_{\lambda \mu}^{r}\right)=c_{p q}^{r} \mathcal{F}_{\lambda \mu}^{p} \xi^{q}, \quad J^{1} u(\xi)\left(\mathcal{S}_{\lambda \mu}^{r}\right)=c_{p q}^{r} \mathcal{S}_{\lambda \mu}^{p} \xi^{q}+c_{p q}^{r} a_{\mu}^{p} \partial_{\lambda} \xi^{q}+\partial_{\lambda} \partial_{\mu} \xi^{r}
$$

Therefore, one can choose the gauge conditions

$$
g^{\lambda \mu} S_{\lambda \mu}^{r}(x)-\alpha^{r}(x)=\frac{1}{2} g^{\lambda \mu}\left(\partial_{\lambda} a_{\mu}^{r}(x)+\partial_{\mu} a_{\lambda}^{r}(x)\right)-\alpha^{r}(x)=0,
$$

which are the familiar generalized Lorentz gauge. The corresponding second-order differential operator (49) reads

$$
M_{s}^{r} \xi^{s}=g^{\lambda \mu}\left(\frac{1}{2} c_{p q}^{r}\left(\partial_{\lambda} a_{\mu}^{r}+\partial_{\mu} a_{\lambda}^{r}\right) \xi^{q}+c_{p q}^{r} a_{\mu}^{p} \partial_{\lambda} \xi^{q}+\partial_{\lambda} \partial_{\mu} \xi^{r}\right) .
$$

Passing to the Euclidean space and repeating the quantization procedure in Section 5, we come to the generating functional

$$
\begin{aligned}
Z= & \mathcal{N}^{-1} \int \exp \left\{\int \left(p_{r}^{\lambda \mu} \mathcal{F}_{\lambda \mu}^{r}-a_{G}^{m n} g_{\mu \nu} g_{\lambda \beta} p_{m}^{\mu \lambda} p_{n}^{\nu \beta} \sqrt{|g|}-\right.\right. \\
& \frac{1}{8} a_{r s}^{G} g^{\alpha \nu} g^{\lambda \mu}\left(\partial_{\alpha} a_{\nu}^{r}+\partial_{\nu} a_{\alpha}^{r}\right)\left(\partial_{\lambda} a_{\mu}^{s}+\partial_{\mu} a_{\lambda}^{s}\right)-g^{\lambda \mu} \bar{c}_{r}\left(\frac{1}{2} c_{p q}^{r}\left(\partial_{\lambda} a_{\mu}^{r}+\partial_{\mu} a_{\lambda}^{r}\right) c^{q}+c_{p q}^{r} a_{\mu}^{p} c_{\lambda}^{q}+c_{\lambda \mu}^{r}\right) \\
& \left.\left.+i J_{r}^{\mu} a_{\mu}^{r}+i J_{\mu \lambda}^{r} p_{r}^{\mu \lambda}\right) \omega\right\} \prod_{x}[d \bar{c}][d c][d p(x)][d a(x)] .
\end{aligned}
$$

Its integration with respect to momenta restarts the familiar generating functional of gauge theory.

\section{REFERENCES}

Castrillón Lòpez, M. and Marsden, J. (2003). Journal of Geometry and Physics 48, 52.

Echeverría-Enríquez, A., Muñoz-Lecanda, M. and Roman-Roy, N. (2000). Journal of Mathematical Physics 41, 7402.

Echeverría-Enríquez, A., López, G., Marin-Solano, J., Muñoz-Lecanda, M. and Roman-Roy, N. (2004). Journal of Mathematical Physics 45, 360.

Forger, M., Paufler, C. and Roemer, H. (2003). Review on Mathematical Physics 15, 705. 
Giachetta, G., Mangiarotti, L. and Sardanashvily, G. (1997). New Lagrangian and Hamiltonian Methods in Field Theory, World Scientific, Singapore.

Giachetta, G., Mangiarotti, L. and Sardanashvily, G. (1999). Journal of Physics A: Mathematical and General 326629.

Hélein, F. and Kouneiher, J. (2002). Journal of Mathematical Physics 43, 2306.

Gotay, M. (1991). Differential Geometry and Its Application 1, 375.

Kanatchikov, I. (1999). Reports on Mathematical Physics 43, 157.

Sardanashvily, G. (1994). International Journal of Theoretical Physics 33, 2365. 